\title{
Transition from Tonks-Girardeau gas to super-Tonks-Girardeau gas as an exact many-body dynamics problem
}

\author{
Shu Chen, ${ }^{1}$ Liming Guan, ${ }^{1}$ Xiangguo Yin, ${ }^{1}$ Yajiang Hao,${ }^{2}$ and Xi-Wen Guan ${ }^{3}$ \\ ${ }^{1}$ Institute of Physics, Chinese Academy of Sciences, Beijing 100190, China \\ ${ }^{2}$ Department of Physics, University of Science and Technology Beijing, Beijing 100083, China \\ ${ }^{3}$ Department of Theoretical Physics, Research School of Physics and Engineering, \\ Australian National University, Canberra ACT 0200, Australia
}

(Dated: November 2, 2018)

\begin{abstract}
We investigate transition of a one-dimensional interacting Bose gas from a strongly repulsive regime to a strongly attractive regime, where a stable highly excited state known as the super Tonks-Girardeau gas was experimentally realized very recently. By solving exact dynamics of the integrable Lieb-Liniger Bose gas, we demonstrate that such an excited gas state can be a very stable dynamic state. Furthermore we calculate the breathing mode of the super Tonks-Girardeau gas which is found to be in good agreement with experimental observation. Our results show that the highly excited super Tonks-Girardeau gas phase can be well understood from the fundamental theory of the solvable Bose gas.
\end{abstract}

PACS numbers: 03.75.Kk, 05.30.Jp, 05.30.-d

Introduction. - Recent experimental advances with ultracold atoms have opened up a new avenue for the study of one-dimensional (1D) strongly correlated many-body systems which continue to inspire significant developments in physics [1-4]. Exquisite tunability of 1D effective interacting strength via Feshbach resonance or the confinement-induced resonance [5-7] allows the simulation of low-dimensional quantum gases with arbitrary interaction strength. The experimental realization of Tonks-Girardeau (TG) gases [3, 4] has provided a direct test on the fermionization of Bose gas in the strongly interacting limit [8, 9]. Very recently, Haller et al [10] have made a new experimental breakthrough on the realization of the stable highly excited gas-like phase called the super-Tonks-Girardeau (STG) gas, which was predicted theoretically [11] and verified from the integrable interacting Bose gas with attractive interactions [12]. Their experimental results have stimulated further theoretical study of such novel states with strong correlations in 1D many-body systems [13], which was also found theoretical correspondence in a strongly attractive Fermi gas without polarization [14].

In general, experimental realization of a stable excited state is difficult and a little counter-intuition since it has no analogue in traditional solid state systems, where a pure excited state is not stable due to the energy dissipation between the system and the environment. The experimental progress [10] opens the door for searching novel quantum states in quantum gases and studying physical properties of stable excited phases, which provides a promising new area of activity in cold atoms. However, the metastability, dynamics and statistical signature of the excited states are still far from understood. Current theoretical understanding of the STG gas is based on intuitive explanation that the STG gas inherits hard core behavior from the repulsive TG gas which prevents the collapse to its true cluster ground state (GS) [11 17]. As a matter of fact, the STG gas is obtained from the TG gas by a sudden switch of interactions, this process is in principle a dynamics problem for the interacting boson model. Therefore a comprehensive understanding of the STG gas is highly desirable and it is also important to show that such a stable state really follows from the fundamental quantum dynamics of an exactly solvable model.

In this work, we demonstrate how the GS of the repulsive TG gas translates to the highly excited state of the strongly attractive TG gas through a sudden switch of interactions. We also show that the highly excited STG phase is stable and the transition rate to the GS is almost completely suppressed in the strongly interacting regime. Furthermore, we calculate the energy of the STG gas by solving the exact Bethe ansatz equations (BAEs) and determine the collective excitation modes of the STG gas with the help of the local density approximation (LDA), which agrees quantitatively with the experimental data.

Interacting boson model. - We consider a quasi-1D system with $N$ bosons tightly confined in an elongated trap which is described by an effective 1D Hamiltonian

$$
H=\sum_{i=1}^{N}-\frac{\hbar^{2}}{2 m} \frac{\partial^{2}}{\partial x_{i}^{2}}+g \sum_{i<j} \delta\left(x_{i}-x_{j}\right),
$$

with $g=-2 \hbar^{2} /\left(m a_{1 d}\right)$ being the effective $1 \mathrm{D}$ interaction strength and $a_{1 d}$ the effective $1 \mathrm{D}$ scattering length [5, 6]. As a simple but fundamental integrable interacting boson model, the exact results for the Lieb-Liniger (LL) model (1) play an important role in understanding quantum statistical and many-body correlation effects of a $1 \mathrm{D}$ bosonic quantum gas [9, 18 21]. The model (11) is exactly solved by the Bethe ansatz method with eigenfunctions taken as superpositions of plane waves over all permutations of the momenta

$$
\Psi\left(x_{1}, \cdots, x_{N}\right)=\sum_{P} A_{P} e^{\mathrm{i} \sum_{j} k_{P_{j}} x_{j}},
$$




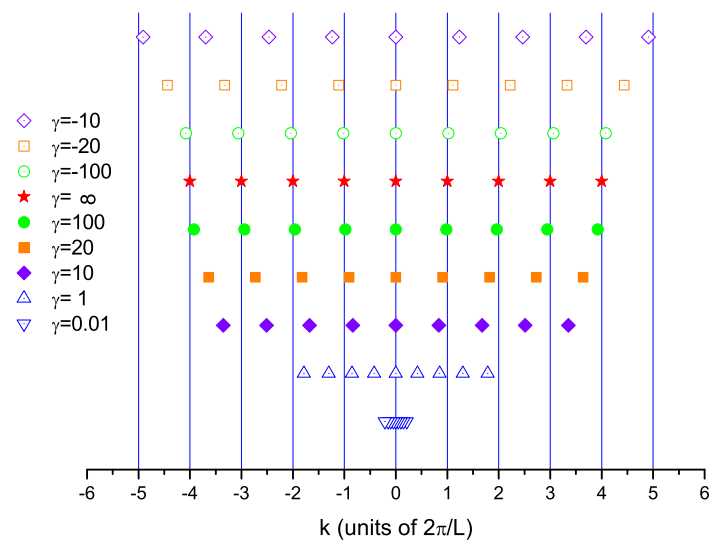

FIG. 1: (color online) Quasi-momentum distributions for the ground state of the repulsive and the STG gas phase of the attractive Bose gas with different values of $\gamma$.

where the wave functions are defined in the domain $x_{1}<x_{2}<\cdots<x_{N}$ and the coefficients $A_{P}=$ $(-1)^{P} \prod_{j<l}^{N} \frac{i k_{p_{l}}-i k_{p_{j}}+c}{\sqrt{\left(k_{p_{l}}-k_{p_{j}}\right)^{2}+c^{2}}}$ are functions of the twoparticle scattering phase shifts with $(-1)^{P}= \pm 1$ for odd or even $P$. The quasi-momenta $k_{j}$ are determined by the BAEs [9]

$$
\exp \left(\mathrm{i} k_{j} L\right)=-\prod_{l=1}^{N}\left(\frac{k_{j}-k_{l}+\mathrm{i} c}{k_{j}-k_{l}-\mathrm{i} c}\right) .
$$

Here the coupling constant $g=\hbar^{2} c / m$ with interaction strength $c=-2 / a_{1 \mathrm{~d}}$ determined by the effective $1 \mathrm{D}$ scattering length $a_{1 \mathrm{~d}}$. The total momenta of the system is given by $K=\sum_{j=1}^{N} k_{j}$ and the eigenenergies are given by $E=\frac{\hbar^{2}}{2 m} \sum_{j=1}^{N} k_{j}^{2}$.

For repulsive interaction $c>0$, all solutions $k_{j}$ of (3) with $j=1, \cdots, N$ are real numbers. Alternatively the solutions follow by solving the log form

$$
k_{j} L=2 \pi I_{j}-\sum_{i=1}^{N} 2 \arctan \left[\left(k_{j}-k_{i}\right) /|c|\right]
$$

of the BAEs. The GS solution corresponds to $I_{j}=(N+$ $1) / 2-j$. On the other hand, for attractive interaction $c<0$, the GS solution to the BAEs follows from the $N$ string solution $k_{j}=\lambda_{j}+\mathrm{i}(N+1-2 j)\left(c / 2+\delta_{j}\right)$, where $j=1, \cdots, N$ and $\delta_{j}$ are small deviations which fall off exponentially to zero as $c \rightarrow-\infty$. Correspondingly, the GS is described by a cluster state [15] with the energy $E_{0}=-\frac{1}{12} c^{2} N\left(N^{2}-1\right)$.

STG phase - Although the GS solution corresponds to the $N$-string solution, the BAEs (3) still have real solutions even for $c<0$, which however correspond to some highly excited state of the attractive Bose gas and can be determined by solving the BAEs

$$
k_{j} L=2 \pi I_{j}+\sum_{i=1}^{N} 2 \arctan \left[\left(k_{j}-k_{i}\right) /|c|\right] .
$$

For the solutions with $I_{j}=(N+1) / 2-j$, the corresponding state is just the STG gas state [12]. In the strongly attractive interaction limit $(c \rightarrow-\infty)$, the quasimomentum distribution of real roots determined from (5) reduces to the free Fermi distribution with a minimum separation between two quasimomenta $2 \pi / L$. As the attraction strength decreases, the gas-like highly excited state has a stronger anyonic-like pressure than the Fermi pressure. For strongly attractive interaction $(c \rightarrow-\infty)$, the energy of the STG gas state [12] is given by $E_{S T G}=$ $\frac{\hbar^{2}}{2 m} \frac{1}{3} N\left(N^{2}-1\right) \frac{\pi^{2}}{L^{2}}\left[1+\frac{4 N}{L|c|}+\frac{12 N^{2}}{L^{2}|c|^{2}}+\cdots\right]$, which is continuously connected to the GS energy of the TG gas $E_{T G}=\frac{\hbar^{2}}{2 m} \frac{1}{3} N\left(N^{2}-1\right) \frac{\pi^{2}}{L^{2}}\left[1-\frac{4 N}{L|c|}+\frac{12 N^{2}}{L^{2}|c|^{2}}-\cdots\right]$ as $|c| \rightarrow \infty$ [8].

Quench dynamics - Suppose that the initial state $\left|\Psi_{i n}(X, t=0)\right\rangle=\left|\psi_{0}(X, c)\right\rangle$ is prepared in the strongly repulsive limit $c \gg 1$, after a quick switch from the repulsive regime to the attractive regime with $c^{\prime}<0$, the wavefunction $|\Psi(X, t)\rangle=e^{\mathrm{i} H t}\left|\Psi_{i}(X, c)\right\rangle$ can be calculated via

$$
|\Psi(X, t)\rangle=\sum_{n=0}^{\infty} e^{i E_{n} t} c_{n}\left|\psi_{n}\left(X, c^{\prime}\right)\right\rangle,
$$

where $c_{n}=\left\langle\psi_{n}\left(X, c^{\prime}\right) \mid \psi_{0}(X, c)\right\rangle$ with $\psi_{n}\left(X, c^{\prime}\right)$ representing the $n$-th eigenstate of the LL model with attractive interaction strength $c^{\prime}$ and $X$ the abbreviation of $x_{1}, \cdots, x_{N}$. From (16), it is straightforward that the probability for the state after quench staying in a STG phase is given by $\left|\left\langle\psi_{S T G}\left(X, c^{\prime}\right) \mid \psi_{0}(X, c)\right\rangle\right|^{2}$, which also represents the transition probability from an initial TG phase to the final STG phase. The wavefunction $\psi_{S T G}$ is determined by the Bethe ansatz wave function (2) with $k_{j}$ determined by the solutions of (5) with $I_{j}=(N+1) / 2-j$. Similarly, the transition probability from the initial TG state to the cluster state is given by $\left|\left\langle\psi_{\text {cluster }}\left(X, c^{\prime}\right) \mid \psi_{0}(X, c)\right\rangle\right|^{2}$. Since the Bethe ansatz wave functions $\psi_{n}(X, c)$ can be exactly determined by solving BAEs, in principle we can calculate the transition probabilities exactly.

For simplicity, we first consider the case with $c^{\prime}=-c$, i.e., the system is initially in the GS of the LL gas with $c>0$, and then suddenly switch to the attractive regime with strength $-c$. We note that the BAE solutions for the repulsive LL gas and that for the STG gas correspond to the same set of $I_{j}$ according to Eqs. (4) and (50). In the limit $c=-c^{\prime} \rightarrow \infty$, the solutions are given by $k_{j}=I_{j} 2 \pi / L$, i.e. they are exactly the same. In Fig. 1 , for particle number $N=9$, we show the BAE solutions for the repulsive LL gas and the STG gas with different values of $\gamma=c / \rho$. In the strongly interacting regime, i.e. $|\gamma| \gg 1$, the quasi-momentum distributions for the repulsive LL gas and the STG gas approach the free fermion "orbitals" from different sides. 


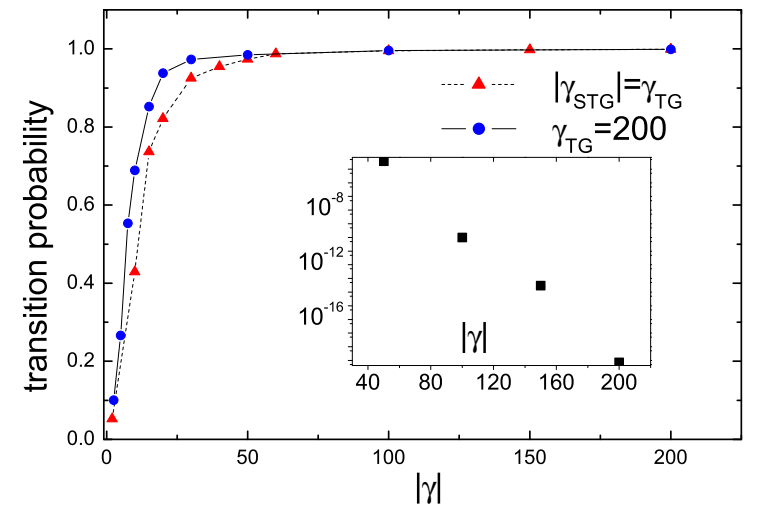

FIG. 2: (color online) Transition probabilities from the TG gas to STG phase. Inset: the transition probabilities from TG gas to the cluster state.

As $|\gamma|$ decreases, the deviation from the free-fermion distribution become more obvious. From the change of momentum distribution, we can expect that the overlap between the GS wavefunction of the strongly repulsive LL gas and the eigen-wavefunction for a STG gas, i.e., $\left|\left\langle\psi_{S T G}(X,-c) \mid \psi_{0}(X, c)\right\rangle\right|$, is close to 1 for very large $|\gamma|$ and exactly 1 when $|\gamma| \rightarrow \infty$.

To see how the transition rate changes with respect to $\gamma$, we calculate the overlap of the wavefunctions between the repulsive and attractive regimes. In order to conceive the signature of the overlap between the two regimes, we consider a system with $N=4$ and calculate the transition rate from the initial repulsive LL gas to the STG gas with different $|\gamma|$. In Fig. 2, we show the transition rate from an initial repulsive gas with $\gamma=200$, to a final STG phase with different values $\gamma<0$. As expected, the transition probability to the STG phase is close to 1 after switching to the strongly attractive regime whereas the probability for dynamically falling into the cluster state is almost completely suppressed. However, when the system is switched into the weakly attractive regime, the STG phase is no longer stable and the probability for falling into the cluster state increases quickly. The transition rate for the larger system does not change qualitatively, however the calculation for a large system is a very time-consuming task due to the calculation of multidimensional integrals. Our calculations give a clear signature of the metastable STG gas against collapsing into the cluster state.

Properties for the STG gas-As shown in the above calculation, the transition rate to the STG phase is very high in the strongly interacting regime. Therefore such a highly excited state with a stronger pressure than the Fermi pressure could be reached through switching the interaction from strong repulsion into strong attraction. We note that such pressure is inherited from the repulsive gas, i.e. transmutes the statistical kinetic energy into the attractive STG phase. In the thermodynamic limit with

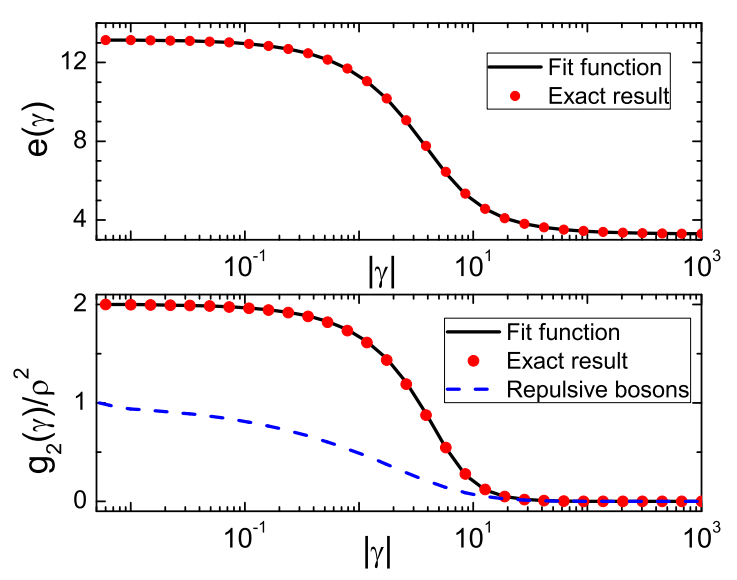

FIG. 3: $e(\gamma)$ and $g_{2}(\gamma)$ versus $|\gamma|$.

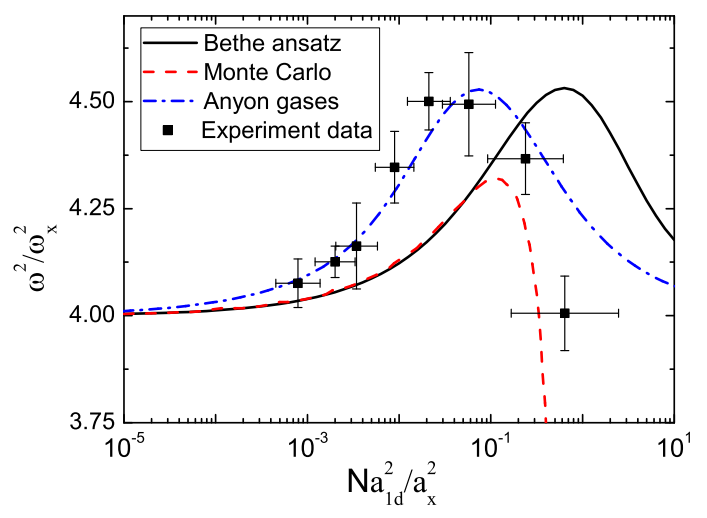

FIG. 4: (color online) Breathing mode of the STG gas. The experiment data with error bars and Monte Carlo result are reproduced from Fig. 3a of Ref. [10].

$N, L \rightarrow \infty$, the eigen energy of the STG phase can be represented in the integral form: $E_{S T G} / L=\frac{\hbar^{2}}{2 m} \int k^{2} \rho(k) \frac{d k}{2 \pi}$ and $\rho(k)=\frac{1}{2 \pi}-\frac{1}{2 \pi} \int_{-Q}^{Q} \frac{2|c| \rho\left(k^{\prime}\right)}{c^{2}+\left(k-k^{\prime}\right)^{2}} d k^{\prime}$, where the integration limit $Q$ is determined by $\rho=\int_{-Q}^{Q} \rho(k) d k$. By making replacement of variables $k=Q x, c=Q \lambda$ and $g(x)=\rho(Q x)$ according to [9], the energy per atom can be expressed as $\epsilon(\rho)=\frac{\hbar^{2}}{2 m} \rho^{2} e(\gamma)$, which can be obtained by solving the system of equations

$$
\begin{aligned}
& e(\gamma)=\left|\frac{\gamma}{\lambda}\right|^{3} \int_{-1}^{1} g(x) x^{2} d x, \\
& g(x)=\frac{1}{2 \pi}-\frac{1}{2 \pi} \int_{-1}^{1} \frac{2 \lambda g\left(x^{\prime}\right)}{\lambda^{2}+\left(x-x^{\prime}\right)^{2}} d x^{\prime},
\end{aligned}
$$

and $\lambda=|\gamma| \int_{-1}^{1} g(x) d x$. The above equations are very similar to Lieb-Liniger's solutions for the repulsive boson gas except the minus sign in Eq. (8). By numerically solving the integral equation, we can ob- 
tain $e(\gamma)$ and thus the energy density $\epsilon(\rho)$ and the chemical potential $\mu(\rho)=\partial_{\rho}[\rho \epsilon(\rho)]$. For the STG phase in the strongly attractive regime, we find $e(\gamma) \approx$ $\frac{\pi^{2}}{3}\left(1+\frac{4}{|\gamma|}+\frac{12}{|\gamma|^{2}}+\frac{32}{|\gamma|^{3}}\left(1-\frac{\pi^{2}}{15}\right)\right)$.

In Fig.3, we show $e(\gamma)$ for different values of $\gamma$, which is found to be well fitted in the whole attractive regime by a rational function $e(\gamma)=\frac{4 \pi^{2}}{3}\left(1+p_{1}|\gamma|+p_{2} \gamma^{2}+\right.$ $\left.p|\gamma|^{3} / 4\right) /\left(1+q_{1}|\gamma|+q_{2} \gamma^{2}+p|\gamma|^{3}\right)$ with $p_{1}=0.075$, $p_{2}=0.013, q_{1}=0.227, q_{2}=0.034, p=0.004$. We also calculate the local two-particle correlation function $g_{2}=$ $\left\langle\Psi^{\dagger} \Psi^{\dagger} \Psi \Psi\right\rangle$ which can be obtained by $g_{2}(\gamma)=\rho^{2} d e(\gamma) / d \gamma$ [20]. The STG phase exhibits stronger local correlation than the repulsive Bose gas. For the STG gas in a harmonic trap with $V_{e x t}=m \omega_{x}^{2} x^{2} / 2$, we can determine the density distribution of the STG gas using LDA [21, 22].

According to the LDA, one can assume that the system is in local equilibrium at each point $x$ in the external trap. The density distribution of the STG gas is then obtained from the local equation of state $\mu[\rho(x)]=\mu_{0}-V_{\text {ext }}(x)$ under Thomas-Fermi approximation with the normalization condition $\int d x \rho(x)=N$. Using the density distribution, one can then calculate the mean square radius of the trapped STG gas via $\left\langle x^{2}\right\rangle=\int \rho(x) x^{2} d x / N$. Following Ref. [11, 22], we can calculate the frequency of the lowest breathing mode given by $\omega^{2}=-2\left\langle x^{2}\right\rangle /\left(d\left\langle x^{2}\right\rangle / d \omega_{x}^{2}\right)$. Comparing with the Monte Carlo simulations [11], our result (solid line) based on the exact solutions of BAEs has a pronounced peak with a maximum of breathing mode frequency $\omega^{2} / \omega_{x}^{2}$ reaching the value about 4.5 which coincides with the experimental data, see Fig 4 Here the discrepancy between the theoretical prediction from the LL model and the experimental data inspires us to search for a new theoretical framework capable of understanding the quantum dynamics of the excited quantum gas phase.

To this goal, we notice that the resonance regime of switching the interaction from strong repulsion to strong attraction can be identified as anyonic type of interaction $c / \cos (\kappa / 2)[23,24]$. We also understand that strongly in- teracting bosons have lost their bosonic signature due to the transmutation between statistical and dynamical interactions [14]. This naturally suggests us to consider the integrable model of anyons as a fitting model to the experiment on the STG gas. The dynamic process of switching the interaction from strong repulsion to strong attraction may be viewed as the change of quantum statistics from less to more exclusive than Fermi statistics. In order to conceive this nature of the STG gas, we also calculate the breathing mode of the integrable model of anyons with $\kappa=3.93$ greater than the free Fermi case $\kappa=\pi$, see dash-dotted line. The good agreement with experiment data implies that the anyonic signature may relate to the statistical signature of the highly excited state of interacting bosons. However, we notice that in the weak coupling regime the involvement of cluster states of few particles [13] is the main reason for the discrepancy between theoretical prediction and experimental data. When the system is not in the strongly attractive regime, the STG phase is no longer stable, see Fig 4 .

Summary - In summary, we have investigated the transition from the GS of strongly repulsive bosons to the gas-like highly excited state of attractive bosons through a switch of interaction. By solving the quench dynamics problem of the interacting bosons, we have shown that the gas-like excited state is stable in the strongly attractive regime. Our exact result for the frequency of the lowest breathing mode obtained from the BAEs for interacting bosons and anyons are in reasonably good agreement with experimental observation. This suggests a statistical signature of the STG gas which may be viewed as an anyon-like gas.

This work is supported by NSFC under Grants Nos. 10821403 and 10974234, 973 project (China). XWG has been supported by the Australian Research Council. We thank M T Batchelor for helpful discussions and critical reading of the paper.
[1] A. Görlitz, et.al., Phys. Rev. Lett. 87, 130402 (2001).

[2] H. Moritz, et.al., Phys. Rev. Lett. 91, 250402 (2003); T. Stöferle, et.al., ibid. 92, 130403 (2004).

[3] B. Paredes, et. al., Nature 429, 277 (2004).

[4] T. Kinoshita, et. al., Science 305, 1125 (2004).

[5] M. Olshanii, Phys. Rev. Lett. 81, 938 (1998).

[6] T. Bergeman, et. al., Phys. Rev. Lett. 91, 163201 (2003).

[7] S. Sinha et. al., Phys. Rev. Lett. 99, 140406 (2007).

[8] M. D. Girardeau, J. Math. Phys. (N.Y.) 1, 516 (1960).

[9] E. H. Lieb and W. Liniger, Phys. Rev. 130, 1605 (1963).

[10] E. Haller, et. al., Science 325, 1224 (2009).

[11] G. E. Astrakharchik, J. Boronat, J. Casulleras, and S. Giorgini, Phys. Rev. Lett. 95, 190407 (2005).

[12] M. T. Batchelor, M. Bortz, X. W. Guan, N. Oelkers, J. Stat. Mech. (2005) L10001.

[13] M. D. Girardeau et. al., arXiv: 0912.1633.
[14] S. Chen, et. al., arXiv: 0910.1652.

[15] J. B. McGuire, J. Math. Phys. (N.Y.) 5, 622 (1964).

[16] G. E. Astrakharchik, D. Blume , S. Giorgini and B. E. Granger, Phys. Rev. Lett. 92, 030402 (2004).

[17] E. Tempfli, et. al., New J. Phys. 10, 103021 (2008).

[18] M. D. Girardeau, Phys. Rev. Lett. 91, 040401 (2003).

[19] H. Buljan, et. al., Phys. Rev. Lett. 100, 080406 (2008).

[20] D. M. Gangardt and G. V. Shlyapnikov, Phys. Rev. Lett. 90, 010401 (2003).

[21] V. Dunjko, et. al., Phys. Rev. Lett. 86, 5413 (2001).

[22] C. Menotti, et. al., Phys. Rev. A 66, 043610 (2002).

[23] A. Kundu, Phys. Rev. Lett. 83, 1275 (1999).

[24] M. T. Batchelor, et. al., Phys. Rev. Lett. 96, 210402 (2006). 